\title{
Microfluidic immunosensor for rapid and highly-sensitive salivary cortisol quantification
}

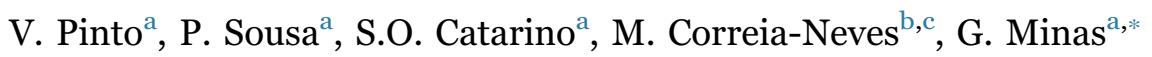 \\ a Microelectromechanical Systems Research Unit (CMEMS-UMinho), University of Minho, Campus de Azurém, 4800-058 Guimarães, Portugal \\ ${ }^{\mathrm{b}}$ Life and Health Sciences Research Institute (ICVS), School of Medicine, Portugal \\ c ICVS/3B's, PT Government Associate Laboratory, University of Minho, Braga/Guimarães, Portugal
}

\section{A R T I C L E I N F O}

\section{Keywords:}

Microfluidic immunosensor

Colorimetric detection

Lab-on-a-chip (LOC)

Poly(dimethylsiloxane)

Salivary cortisol

\begin{abstract}
A B S T R A C T
This paper presents a novel poly(dimethylsiloxane) (PDMS) microfluidic immunosensor that integrates a complementary metal-oxide-semiconductor (CMOS) optical detection system for a rapid and highly-sensitive quantification of salivary cortisol. The simple and non-invasive method of saliva sampling provides an interesting alternative to the blood, allowing a fast sampling at short intervals, relevant for many clinical diagnostic applications. The developed approach is based on the covalent immobilization of a coating antibody (Ab), a polyclonal anti-IgG, onto a treated PDMS surface. The coating Ab binds the capture Ab, an IgG specific for cortisol, allowing its correct orientation. Horseradish peroxidase (HRP)-labelled cortisol is added to compete with the cortisol in the sample, for the capture Ab binding sites. The HRP-labelled cortisol, bonded to the capture Ab, is measured through the HRP enzyme and the tetramethylbenzidine (TMB) substrate reaction. The cortisol quantification is performed by colorimetric detection of HRP-labelled cortisol, through optical absorption at $450 \mathrm{~nm}$, using a CMOS silicon photodiode as the photodetector. Under the developed optimized conditions presented here, e.g., microfluidic channels geometry, immobilization method and immunoassay conditions, the immunosensor shows a linear range of detection between $0.01-20 \mathrm{ng} / \mathrm{mL}$, a limit of detection (LOD) of $18 \mathrm{pg} / \mathrm{mL}$ and an analysis time of $35 \mathrm{~min}$, featuring a great potential for point-of-care applications requiring continuous monitoring of the salivary cortisol levels during a circadian cycle.
\end{abstract}

\section{Introduction}

Nowadays, the physical and psychological chronic stress, originated from stressful daily routine, is known to trigger disorders, such as depression and cardiovascular diseases (Lederbogen et al., 2011). Therefore, a great effort is being made to develop tools for stress monitoring. One of the most important stress biomarkers is the hormone cortisol (Lucassen et al., 2014). Cortisol levels in the body fluids follow a circadian cycle, which have maximum levels in the morning, decreasing during the day and registering a minimum before sleep (Lucassen et al., 2014; Quax et al., 2013). A common feature of maladaptive response to chronic stress is the increased cortisol levels in specific periods or abnormal circadian cycle. Therefore, it is of utmost importance to monitor the cortisol concentration in the human body along the day.

Saliva has emerged as a useful alternative fluid, as compared to blood, for measuring cortisol concentration. Its main advantage relies in an easy and non-invasive collection method, which reduces the stress response and allows a fast sampling at short time intervals, enabling a continuous monitoring of cortisol levels. Moreover, salivary cortisol is present in the free state (biologically active), which is the relevant form for its detection, contrary to what occurs in the blood, where about $90 \%$ of it is bonded to proteins (Gröschl, 2008; Kaushik et al., 2014). However, salivary cortisol levels, which range between 1 and $8 \mathrm{ng} / \mathrm{mL}$ in healthy adults, are up to 100 times smaller than in the blood, requiring highly sensitive and accurate detection methods (Stevens et al., 2008; Yamaguchi et al., 2013).

The traditionally analytical methods for measuring the salivary cortisol levels include high performance liquid chromatography (HPLC) (Chen et al., 2010), fluorometric assay, radioimmunoassay (RIA) (Kaushik et al., 2014), flow immunoassay and enzyme linked immunosorbent assay (ELISA) (Sesay et al., 2013; Tlili et al., 2011). Although these methods provide high sensitivity, they are expensive, time and reagent/sample consuming, involve multiple step reaction, washing processes, and involve the use of radioisotopes, features that are inappropriate for miniature and portable devices (Kaushik et al., 2014; Tlili et al., 2011). Aiming the miniaturization of the device, several antibodies (Ab)-based biosensors (immunosensors) have been

\footnotetext{
* Corresponding author.

E-mail address: gminas@dei.uminho.pt (G. Minas).
} 
reported for cortisol detection based on electrochemical (Tlili et al., 2011) and surface plasmon resonance (SPR) detection techniques (Stevens et al., 2008). Microfluidic devices have emerged as an interesting alternative to those miniaturization efforts, comprising advantages such as efficiency, automation, portability, analysis time and reagents/samples reduction, and above all, a reduction of the overall cost (Bange et al., 2005).

Recently, as an alternative to Ab-based biosensors for cortisol detection, it has been reported the use of aptamers (single-stranded DNA or RNA sequences that recognize specific targets) as an immobilization-free strategy, owing to their simple synthesis and good selectivity and stability (Wei et al., 2015). Although this new strategy presents some potential to be used in microfluidic applications, the $\mathrm{Ab}$ technology is yet preferred for point-of-care devices since it is well established, numerous immunoreagents are commercially available for several analytes and the $\mathrm{Ab}$ have a much stronger binding affinity than aptamers, which means that it is necessary to use more aptamers to do the same task when compared with Ab (Kedzierski, et al., 2012; Hasegawa et al., 2016). In this work the development of a microfluidic immunosensor (Ab-based biosensor) fabricated in poly(dimethylsiloxane) (PDMS) is described. It integrates optical techniques for a rapid and highly-sensitive detection and quantification of salivary cortisol concentration (Fig. 1). The use of a PDMS platform features optical transparency, needed for optical detection; allows fabrication in nanometer features without the need of cleanroom facilities; and allows low-cost for prototyping (Pinto et al., 2014). Moreover, although a paper based microfluidic platform could be advantageous due to a more effective low-cost and simple fabrication (Kakoti, et al., 2015; Li, et al., 2012; Ren et al., 2013), it seems incompatible with the working conditions here due to the very low concentration and small size of the analyte, and the absorbance detection. Usually, a paper-based microfluidic device features limitations regarding the retention or diffusion of the reagents and the sample in the inner highly porous cellulose matrix (dilution), which can block the internal signal in the detection zone, leading to an unsatisfactory detection sensitivity (Ren et al., 2013).

To our knowledge there are no previous reports on a microfluidic immunosensor for salivary cortisol quantification that integrates the immunoassay on a PDMS microfluidic die and the optical absorption as the detection technique. The immunoassay is based on a competitive assay, due to the low molecular weight and the low concentration of the cortisol molecule, enabling low detection limit (Bojorge Ramírez et al., 2009). This competitive assay features an output signal that is inversely proportional to the cortisol concentrations, which is a required feature due to the low cortisol concentrations in saliva. This approach renders this immunosensor an effective device for measuring the salivary cortisol concentration.

\section{Material and methods}

\subsection{Materials}

HRP-labelled cortisol (F-HRP), Hidrocortisone and rabbit anticortisol IgG (capture $\mathrm{Ab}$ ) were obtained from CosmoBio. The polyclonal goat Ab anti-rabbit-IgG (coating Ab), 3,3',5,5'Tetramethylbenzidine (TMB), aminopropyltriethoxysilane (APTES), glutaraldehyde (GA), Tween20 and bovine serum albumin (BSA) were obtained from Sigma-Aldrich. Sylgard 184 silicone elastomer (PDMS) was purchased from Dow Corning and the SU-8 negative photoresist from MicroChem. All other chemicals were obtained from Thermo Fisher Scientific.

A

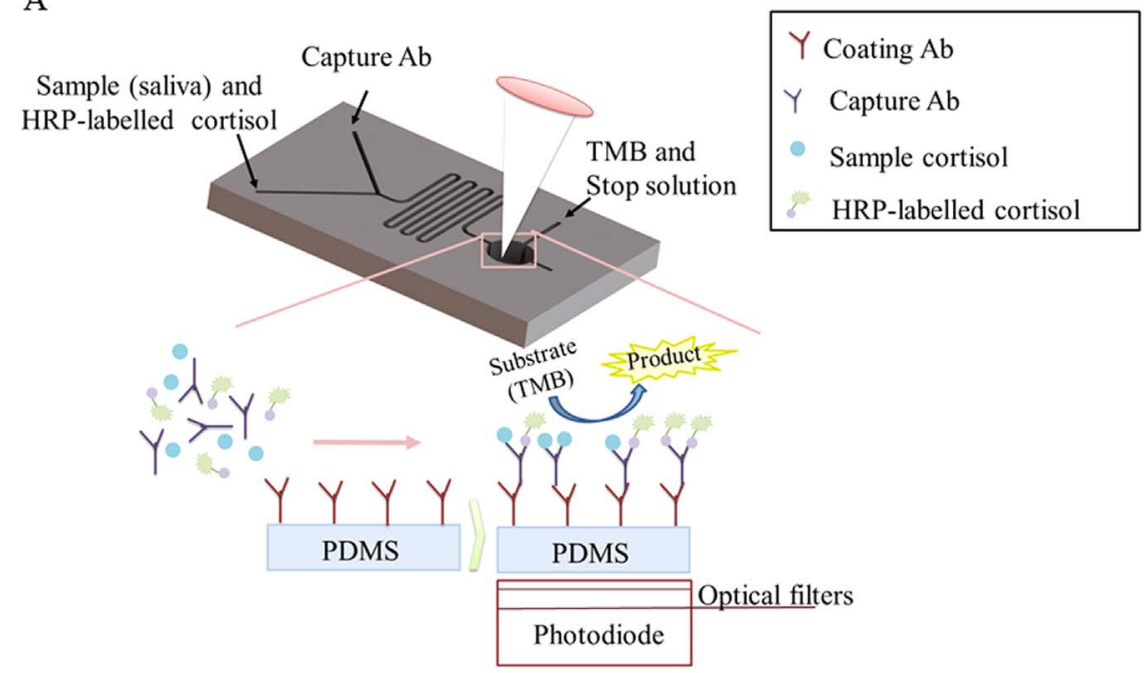

B

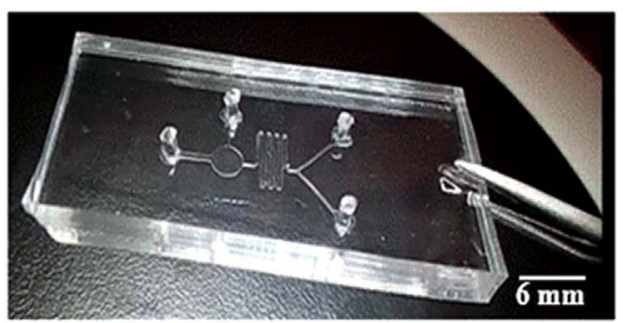

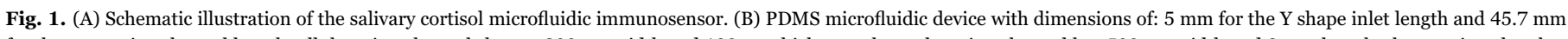

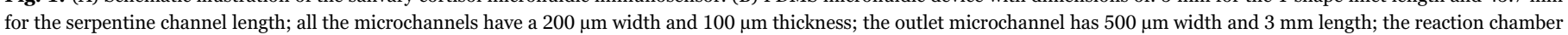
is a cylinder shape with a diameter and thickness of $3 \mathrm{~mm}$. 


\subsection{Apparatus}

The $100 \mathrm{~W}$ Plasma Systems ZEPTO from Diener electronic was used for the PDMS surface modification. Absorption spectra were recorded with a setup that includes a $250 \mathrm{~W}$ quartz tungsten halogen lamp, as light source; a monochromator (Newport 74125); an optical fiber (Newport Standard Grade FS Fiber Optic), used to guide the light trough the sample and into the photodiodes box; and a picoammeter (Keithley 487) to measure the photodiode current. A neMESYS Syringe Pump system with three $5 \mathrm{~mL}$ volume syringes individually controlled by the neMESYS UserInterface was used to control the flow inside the microchannels.

\subsection{Ab immobilization on the PDMS surface}

The competitive assay uses the covalent immobilization of the coating Ab via GA onto APTES-treated PDMS surface (Fig. S1 in the Supplementary material). For that, the PDMS surface was modified by oxygen plasma during $60 \mathrm{~s}$ featuring the - $\mathrm{Si}(\mathrm{OH}) \mathrm{x}$ groups (Chuah et al., 2015). An aqueous solution of $50 \% \mathrm{v} / \mathrm{v}$ APTES in absolute ethanol was immediately added and incubated during $20 \mathrm{~min}$, which reacts with the hydroxyl groups on plasma-treated PDMS and produces amine groups $\left(-\mathrm{NH}_{2}\right)$. These optimal conditions, especially the APTES concentration and the incubation time, were studied by fluorescamine assay and transmittance (Fig. S2 in the Supplementary material). The PDMS samples were washed with absolute ethanol to remove the APTES excess and dried with a nitrogen flow. After this, the APTES-treated PDMS was incubated at $80{ }^{\circ} \mathrm{C}$ in a vacuum oven for $2 \mathrm{~h}$. The $\mathrm{NH}_{2}-$ funcionalized PDMS surface was then reactivated using 2.5\% of GA in PBS for $1 \mathrm{~h}$ at room temperature (RT) and washed with PBS and dried with nitrogen flow. Finally, the coating Ab was immobilized on the PDMS surface by overnight incubation. Atomic force microscopy (AFM) analyses showed a quite uniform distribution of the coating $\mathrm{Ab}$ on PDMS surface proving the efficiency of this immobilization method (Fig. S3 in the Supplementary material).

\subsection{Blocking solution efficiency for non-specific adsorption on PDMS}

Despite the success of the coating Ab immobilization onto APTEStreated PDMS surface, non-specific proteins can also adsorb to the surface leading to false positive results. Therefore, a blocking solution of $0.1 \%$ BSA $+3 \%$ sucrose, prepared in borate buffer at $\mathrm{pH} 7.8$, was added for $4 \mathrm{~h}$ at RT. Afterwards the PDMS was washed with PBSTween 20 and stored at $4{ }^{\circ} \mathrm{C}$ for, at least, 3 weeks until its use. Table S1 in the Supplementary material shows this solution efficiency.

\subsection{Sensitivity analysis}

The calibration curves of the immunoassay (dependence of absorbance at $450 \mathrm{~nm}$ on HRP-labelled cortisol concentration) were obtained by plotting the absorbance against the logarithm of cortisol standards concentrations ( $0-1000 \mathrm{ng} / \mathrm{mL}$ ) using Origin 8.0. The curve was fitted using a nonlinear four parameters logistic calibration plot and defined by (Sesay et al., 2013):

$\mathrm{y}=\frac{(1-a)}{1+\left(\frac{x}{c}\right)^{b}}+\mathrm{d}$

where $a$ and $d$ are the asymptotic maximum and minimum values, respectively; $c$ is the value at the inflection point; $b$ is the slope; $y$ is the absorbance and $x$ is the logarithm of the cortisol concentration. The sensitivity of the immunoassay may be defined as the limit of detection (LOD), which is defined as the smallest concentration of cortisol that is able to produce a signal that can be distinguished from the signal obtained in the absence of that analyte (Armbruster and Pry, 2008). To make a direct comparison between the various calibration curves, the absorbance data was normalized. This transformation was performed by the values of $\% \mathrm{~A} / \mathrm{A} 0$, where $\mathrm{A}$ is the sample absorbance and $\mathrm{A} 0$ is the absorbance at zero concentration.

\section{Results and discussion}

\subsection{Immunoassay optimization for salivary cortisol on PDMS wells}

In order to find the optimal conditions of the complete immunoassay towards high sensitivity with low sample and reagents volumes, optimization assays were performed with different concentrations of the reagents (coating $\mathrm{Ab}$, capture $\mathrm{Ab}$ and HRP-labelled cortisol), as well as with diverse incubation periods. The optimization was initially performed in rectangular PDMS wells $(4 \mathrm{~mm}$ length $\times 3 \mathrm{~mm}$ width $\times 3 \mathrm{~mm}$ height) at RT. Firstly, the measurements were made with no standard cortisol $(0 \mathrm{ng} / \mathrm{mL})$ to obtain the maximum absorbance signal. In this step several coating Ab concentrations $(2 \mu \mathrm{g} / \mathrm{mL}, 10 \mu \mathrm{g} / \mathrm{mL}$ and $20 \mu \mathrm{g} / \mathrm{mL})$ and capture $\mathrm{Ab}(1: 10000,1: 25000,1: 50000,1: 100000$, $1: 200000 ; 1: 500000,1: 1000000$ and $1: 2000000)$ were used, with a fixed concentration of HRP-labelled cortisol (1:25000). To find the optimal concentration of HRP-labelled cortisol, it was done, simultaneously, a study using several HRP-labelled cortisol dilutions (1:10000, $1: 25000,1: 50000,1: 100000)$ where a fixed concentration of coating $\mathrm{Ab}$ $(10 \mu \mathrm{g} / \mathrm{mL})$ and capture $\mathrm{Ab}(1: 50000)$ were used taking into consideration the previous results of this work.

The optimal concentration extrapolated from our results was $10 \mu \mathrm{g} /$ $\mathrm{mL}$ for the coating $\mathrm{Ab}$ (see Fig. 2A). Despite the absorbance signal of $20 \mu \mathrm{g} / \mathrm{mL}$ being slightly higher, $10 \mu \mathrm{g} / \mathrm{mL}$ was chosen since it provides almost the same absorbance signal and it allows reagents parsimony. The optimal dilution of capture $\mathrm{Ab}$ and HRP-labelled cortisol were determined (1:50000 and 1:25000, respectively), with absorbance values of 1.0, as shown in Fig. $2 \mathrm{~A}$ and $\mathrm{B}$, leading to stable results.

To determine the best reaction time of the capture Ab with HRPlabelled cortisol and standard cortisol, $10 \mu \mathrm{L}$ of each of these reagents were incubated in the PDMS wells, pre-functionalized with coating capture $\mathrm{Ab}$ for $30 \mathrm{~min}, 1 \mathrm{~h}, 2 \mathrm{~h}$, and $3 \mathrm{~h}$. Then, the PDMS wells were washed with PBS-Tween 20 and $20 \mu \mathrm{L}$ of TMB were added for $10 \mathrm{~min}$. Finally, $10 \mu \mathrm{L}$ of stop solution was added and the absorbance measured at $450 \mathrm{~nm}$. The incubation time was defined as $1 \mathrm{~h}$ (Fig. 2C) since the experimental results showed a stabilized absorbance maximum value.

\subsection{Sensitivity of the salivary cortisol analysis on PDMS wells}

The assays for obtaining the calibration curve (Fig. 2D) were performed in the reported Ab immobilization on the PDMS wells, which have previously been pre-functionalized with coating $\mathrm{Ab}$ at a concentration of $10 \mu \mathrm{g} / \mathrm{mL}$. In these wells it was added: $10 \mu \mathrm{L}$ of capture $\mathrm{Ab}$ diluted in borate buffer with the ratio $1: 50000 ; 10 \mu \mathrm{L}$ of HRP-labelled cortisol diluted in borate buffer with the ratio 1:25000; and $10 \mu \mathrm{L}$ of standard cortisol at different concentrations, from 0.1 to $1000 \mathrm{ng} / \mathrm{mL}$ Then, the PDMS wells mixtures were stirred, manually, during $1 \mathrm{~min}$ to promote the mixture of the three reagents and incubated for $1 \mathrm{~h}$. After this time, the PDMS wells were washed with $30 \mu \mathrm{L}$ of PBS-Tween 20 and $20 \mu \mathrm{L}$ of TMB solution and incubated at RT for $10 \mathrm{~min}$. Finally, $10 \mu \mathrm{L}$ of sulfuric acid ( $2 \mathrm{M}$ ) was added to stop the reaction and after that the optical absorption were measured. It was used a low concentration of sulfuric acid (2 M) and short interaction time within microfluidic device and thus, no inconvenience effect was observed in the PDMS. The obtained calibration curve for the optimized immunoassay showed a LOD of $10 \mathrm{pg} / \mathrm{mL}$ and a working range of $0.01-20 \mathrm{ng} / \mathrm{mL}$ (Fig. 2D). These results lead to the implementation of the microfluidic immunoassay.

\subsection{Selectivity of the salivary cortisol analysis on PDMS wells}

In order to verify the specificity of the immunoassay, e.g., if any 


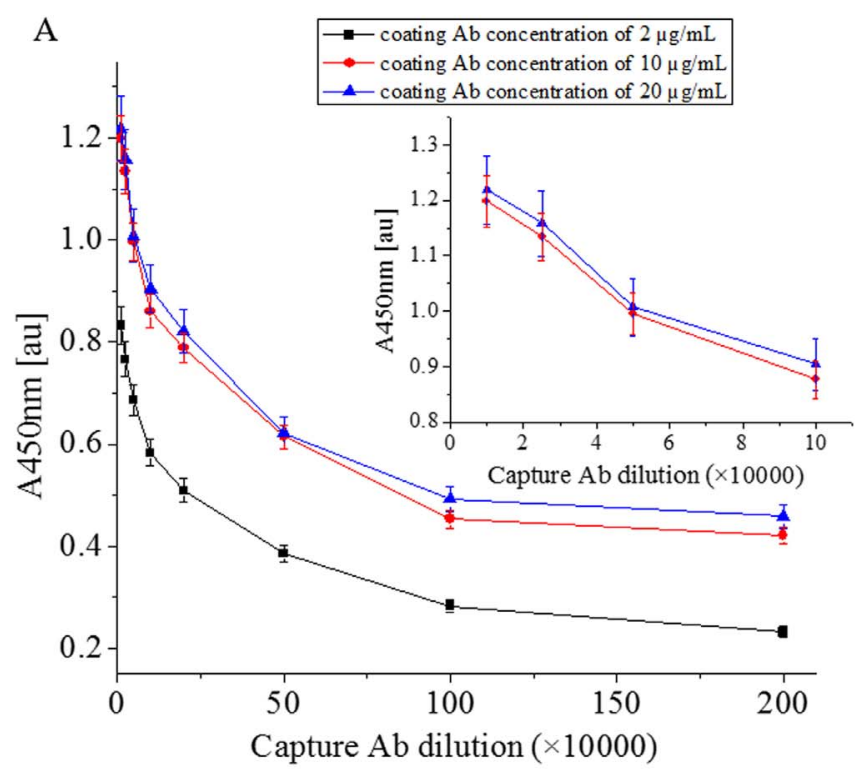

B

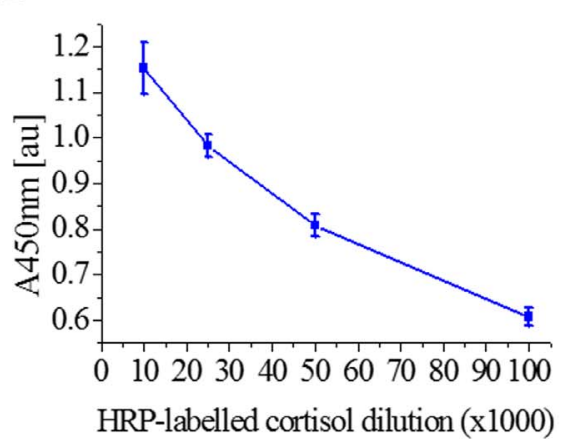

C

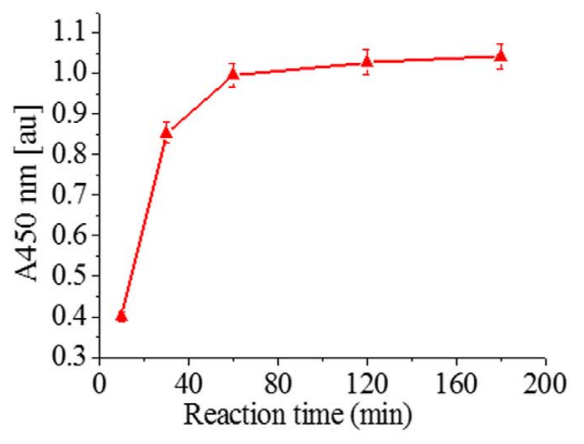

E

$\mathrm{D}$

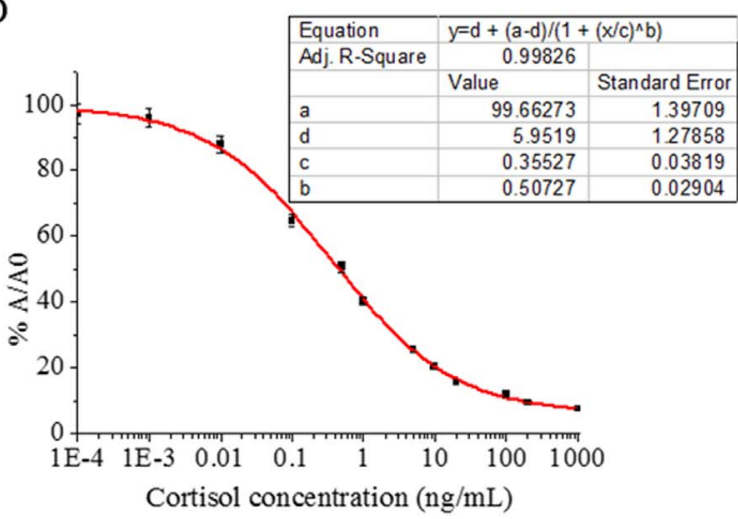

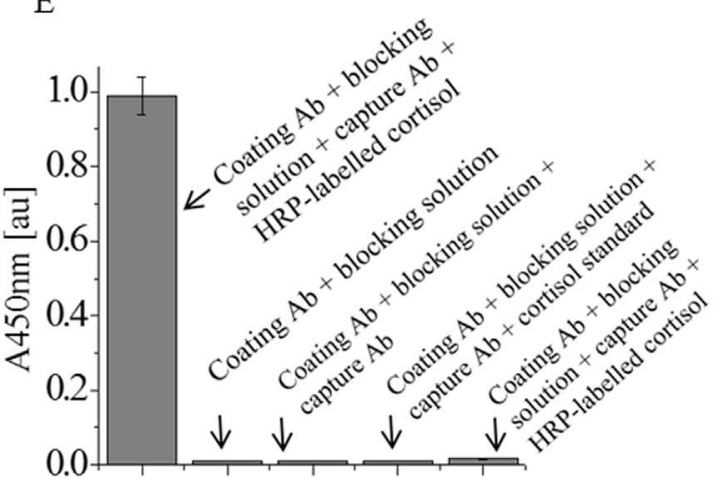

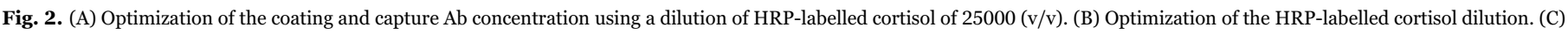

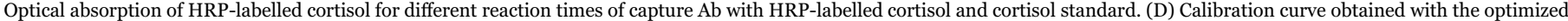

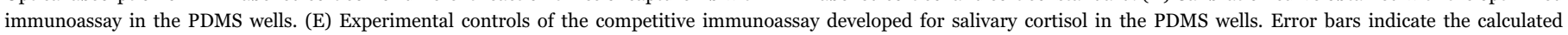
standard deviation $(n=3)$ and maximum standard deviations of (A) $5.1 \%$, (B) $4.7 \%$, (C) $3.6 \%$ and (D) $3.2 \%$ were obtained.

reagents (buffer, blocking solution, coating and capture $\mathrm{Ab}$ ) interfered in the previous absorbance measurements leading to incorrect cortisol concentration results, it was performed absorbance measurements, at $450 \mathrm{~nm}$, considering these reagents alone. The experimental results, presented in Fig. 2E, show that there was not significant interference due to the extremely low absorbance obtained for those reagents in comparison with the test that includes capture Ab and HRP-labelled cortisol, where it is observed the maximum absorbance.

\subsection{Microfluidic device design and fabrication}

After optimizing the immunoassay on PDMS wells, the microfluidic device was designed. It must include two inlets and a channel to mix the fluids before reaching the reaction chamber. The best microchannels geometry and length for an efficient mixing were evaluated through finite elements numerical simulations using COMSOL Multiphysics software. This study aimed to determine if the channels allowed the complete fluids mixture before reaching the reaction 


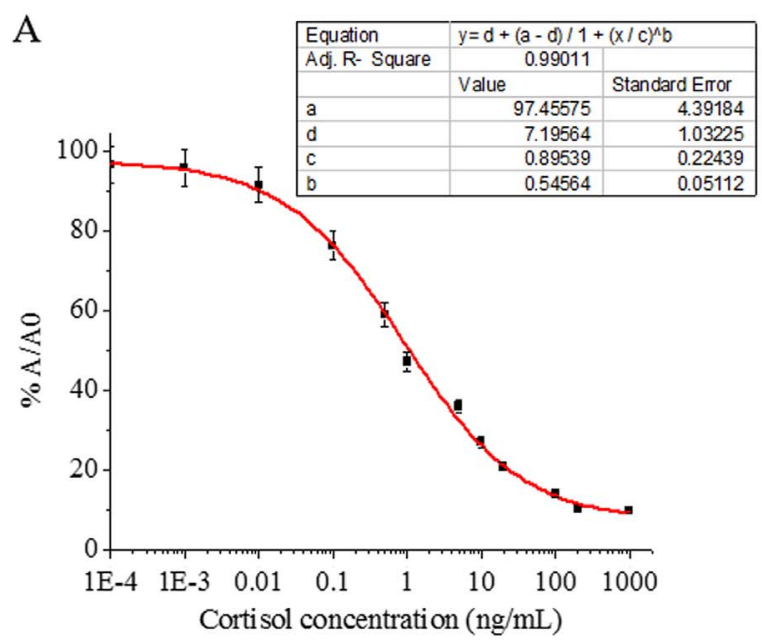

$\mathrm{B}$

\begin{tabular}{|c|c|c|}
\hline & $\begin{array}{l}\text { Microfluidic for } \\
\text { device } \\
\text { salivary cortisol } \\
\text { with photodiode } \\
\text { with active area } \\
1 \times 1 \mathrm{~mm}\end{array}$ & $\begin{array}{c}\text { ELISA } \\
\text { conventional }\end{array}$ \\
\hline $\begin{array}{c}\text { Detection limit } \\
(\mathrm{pg} / \mathrm{mL})\end{array}$ & 18 & 315 \\
\hline $\begin{array}{c}\text { Working range } \\
(\mathrm{ng} / \mathrm{mL})\end{array}$ & $0.01-20$ & $1-500$ \\
\hline $\begin{array}{c}\text { Total assay } \\
\text { duration (h) }\end{array}$ & $\sim 0.58$ & $\sim 14$ \\
\hline $\begin{array}{c}\text { Quantity reagent } \\
(\mu \mathrm{L})\end{array}$ & $5-20$ & $75-200$ \\
\hline $\begin{array}{c}\text { Biosensor } \\
\text { miniaturization }\end{array}$ & Yes & No \\
\hline
\end{tabular}

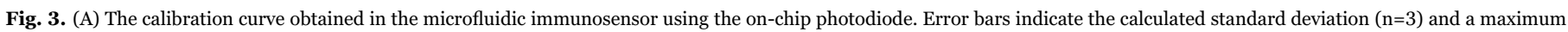
standard deviation of $4.8 \%$ was obtained. (B) Analytical performance of the microfluidic immunosensor in comparison with the conventional gold-standard ELISA method.

chamber. Thus, two different geometries have been designed and simulated: one Y-shaped straight channel with $20 \mathrm{~mm}$ length and $200 \mu \mathrm{m}$ width, as detailed in Tables S2 and S3 in the Supplementary material. The simulation results (Fig. S4, Supplementary material) demonstrated that the serpentine microchannels are the most suitable option for the desired application. This geometry allows a longest flow length, essential to assure the complete mixture of fluids by convectiondiffusion phenomena, maintaining the small dimensions of the microfluidic device.

The fabrication of the PDMS microfluidic device was performed by a soft lithography technique using a SU-8 mold for the microfluidic channel and by micromachining using an acrylic mold for the reaction chamber that was polished off to remove the roughness. Details of the SU-8 molds processing steps can be found in Pinto et al. (2014). The PDMS solution, composed of $1 / 10 \%$ wt of base/curing agent, was poured over the molds and cured at $80^{\circ} \mathrm{C}$ during $2 \mathrm{~h}$. Holes were then punched to provide access to the inlets and to the outlets in the desired location with a syringe tip. In the PDMS reaction chamber it is performed all the optimized immobilization process described in Sections 2.3 and 2.4. Then, it is aligned with the channels slice and closed via $\mathrm{O}_{2}$ plasma. The PDMS serpentine channel for the microfluidic immunosensor application is shown in Fig. 1B. It was designed to be disposable.

\subsection{Optical detection of salivary cortisol in the microfluidic device}

The microfluidic immunosensor was tested using an experimental setup that comprises three subsystems: the microfluidic die; a fluidic pumping control (Nemesys Syringe Pump); and a spectrophotometric system for optical absorption measurements. The immunoassay inside the microfluidic device was performed pumping $5 \mu \mathrm{L}$ of capture $\mathrm{Ab}$ with a flow rate of $2 \mu \mathrm{L} / \mathrm{min}$ in one of the inlets and in the other inlet it was introduced $10 \mu \mathrm{L}$ of HRP-labelled cortisol and cortisol standard ( $5 \mu \mathrm{L}$ of each) with a flow rate of $4 \mu \mathrm{L} / \mathrm{min}$. These last two solutions were incubated in the microfluidic device reaction chamber during $20 \mathrm{~min}$. It should be noticed that, using the same reagents, the incubation time in the PDMS wells was $1 \mathrm{~h}$. This time reduction was due to the serpentine microchannel geometry, to the increase of the contact area in the microchannel interior, as well as to the deduced size of the reaction chamber. Then, the washing solution of PBS-Tween20 was pumped with a flow rate of $4 \mu \mathrm{L} / \mathrm{min}$ during $1 \mathrm{~min}$. The third inlet that leads to the reaction chamber was used to introduce a $10 \mu \mathrm{L}$ of TMB that was incubated for $10 \mathrm{~min}$. After that, this mixture produced a blue color inside the reaction chamber (Fig. S5a in the Supplementary material). Then, it was introduced $5 \mu \mathrm{L}$ of stop solution, which produced a yellow color (Fig. S5b in the Supplementary material) and the optical absorption was measured at $450 \mathrm{~nm}$, using an on-chip silicon p-n junction photodiode with an active area of $1 \times 1 \mathrm{~mm}^{2}$, positioned below the reaction chamber. The on-chip immunosensor calibration curve showed a LOD of $18 \mathrm{pg} / \mathrm{mL}$ and a working range of 0.01-20 ng/mL (Fig. 3A).

Notably, the developed sensor can offer a solution for the detection of cortisol with a significant decrease of the reagents consumption and time (see Fig. 3B), when compared with the conventional goldstandard methods. Furthermore, the microfluidic immunosensor enables cortisol detection down to $18 \mathrm{pg} / \mathrm{mL}$ within about $35 \mathrm{~min}$ analysis time.

These results show the PDMS viability as material for the microfluidic device fabrication, enhancing its main advantages (high optical transparency, easy and stable Ab immobilization on the PDMS surface), when compared to paper-based microfluidic devices, which would have limitations regarding the retention of the analyte, especially when are involved low molecular weight and low cortisol concentration, as in the proposed immunoassay. The presented results also prove the viability of this immunosensor for cortisol quantification with low LOD, using commercial available immunoreagents and through wellestablished methods (Ab-based), when compared to other approaches, e.g. aptamers. Therefore, these results suggest that the developed microfluidic device is promising for quantification of salivary cortisol levels (normal values between $1-8 \mathrm{ng} / \mathrm{mL}$ ).

\section{Conclusions}

This paper reports the development of a novel PDMS microfluidic immunosensor for the determination of salivary cortisol concentration using optical absorption integrated in CMOS. It offers distinct advantages, when compared with the gold-standard methods: (1) decreased reagents consumption (5-20 $\mu \mathrm{L}$ ) and analysis time (35 min); (2) lowcost and easy fabrication; (3) LOD of $18 \mathrm{pg} / \mathrm{mL}$; (4) linear detection range between $0.01-20 \mathrm{ng} / \mathrm{mL}$; (5) point-of-care applications for monitoring salivary cortisol secretion in a circadian cycle.

In the future, the immunosensor can be fabricated with a miniaturized and reusable pumping, mixing and control system that includes the actuation components (e.g. piezoelectric micropumps) integrated in the electronic systems (Rife et al., 2000; Catarino et al., 2014, 2016). To do so one can make use of commercially available compact and lightweight systems, such as micro piezo pumps, specifically designed for microfluidics applications. 


\section{Acknowledgments}

This work was supported by FCT with the reference project UID/ EEA/04436/2013, by FEDER funds through the COMPETE 2020 Programa Operacional Competitividade e Internacionalização (POCI) with the reference project POCI-01-0145-FEDER-006941. V C Pinto thanks the FCT for the SFRH/BD/81526/2011 PhD grant. P J Sousa thanks the FCT for the SFRH/BD/81562/2011 PhD grant. S.O. Catarino thanks the FCT for the SFRH/BPD/108889/2015 grant, supported by national funds from Ministérios da Ciência, Tecnologia e Ensino Superior and by FSE through the POCH - Programa Operacional Capital Humano.

\section{Appendix A. Supplementary material}

Supplementary data associated with this article can be found in the online version at doi:10.1016/j.bios.2016.11.067.

\section{References}

Armbruster, D.A., Pry, T., 2008. Clin. Biochem. Rev. 29 (Suppl 1), S49-S52. Bange, A., Halsall, H.B., Heineman, W.R., 2005. Biosens. Bioelectron. 20 (12), 2488-2503.

Bojorge Ramírez, N., Salgado, A., Valdman, B., 2009. Braz. J. Chem. Eng. 26 (2), $227-249$.

Catarino, S.O., Silva, L.R., Mendes, P.M., Miranda, P.M., Lanceros-Mendez, S., Minas, M., 2014. Sens. Actuators B 205, 206-214.

Catarino, S. O., Pinto, V. C., Sousa, P. J., Lima, R., Miranda, J. M., Minas, G.2016. In:
Engineering in Medicine and Biology Society (EMBC), IEEE Proceedings of the 38th Annual International Conference, 5660-5663

Chen, L.Q., Kang, X.J., Sun, J., Deng, J.J., Gu, Z.Z., Lu, Z.H., 2010. J. Sep. Sci. 33 (15), 2369-2375.

Chuah, Y.J., Kuddannaya, S., Lee, M.H.A., Zhang, Y., Kang, Y., 2015. Biomater. Sci. 3 (2), 383-390.

Gröschl, M., 2008. Clin. Chem. 54 (11), 1759-1769.

Hasegawa, H., Savory, N., Abe, K., Ikebukuro, K., 2016. Molecules 21 (4), 421.

Kakoti, A., Siddiqui, M.F., Goswami, P., 2015. Biomicrofluidics 9 (2), 026502.

Kaushik, A., Vasudev, A., Arya, S.K., Pasha, S.K., Bhansali, S., 2014. Biosens. Bioelectron. 53, 499-512.

Kedzierski, S.U.Z.Y., Khoshnejad, M.A.K.A.N., Caltagirone, G.T., 2012. Bioprocess. J. 11, 46-49.

Lederbogen, F., Kirsch, P., Haddad, L., Streit, F., Tost, H., Schuch, P., Wüst, S., Pruessner, J.C., Rietschel, M., Deuschle, M., 2011. Nature 474 (7352), 498-501.

Li, X., Ballerini, D.R., Shen, W., 2012. Biomicrofluid 6 (1), 011301.

Lucassen, P.J., Pruessner, J., Sousa, N., Almeida, O.F., Van Dam, A.M., Rajkowska, G., Swaab, D.F., Czéh, B., 2014. Acta Neuropathol. 127 (1), 109-135.

Pinto, V.C., Sousa, P.J., Cardoso, V.F., Minas, G., 2014. Micromachines 5 (3), 738-755. Quax, R.A., Manenschijn, L., Koper, J.W., Hazes, J.M., Lamberts, S.W., Van Rossum, E.F., Feelders, R.A., 2013. Nat. Rev. Endocrinol. 9 (11), 670-686.

Ren, K., Zhou, J., Wu, H., 2013. Acc. Chem. Res. 46 (11), 2396-2406.

Rife, J.C., Bell, M.I., Horwitz, J.S., Kabler, M.N., Auyeung, R.C.Y., Kim, W.J., 2000. Sens. Actuators A Phys. 86, 135-140.

Sesay, A.M., Micheli, L., Tervo, P., Palleschi, G., Virtanen, V., 2013. Anal. Biochem. 434 (2), 308-314.

Stevens, R.C., Soelberg, S.D., Near, S., Furlong, C.E., 2008. Anal. Chem. 80 (17), 6747-6751.

Tlili, C., Myung, N.V., Shetty, V., Mulchandani, A., 2011. Biosens. Bioelectron. 26 (11), 4382-4386.

Wei, Y., Chen, Y., Li, H., Shuang, S., Dong, C., Wang, G., 2015. Biosens. Bioelectron. 63, 311-316.

Yamaguchi, M., Matsuda, Y., Sasaki, S., Sasaki, M., Kadoma, Y., Imai, Y., Niwa, D., Shetty, V., 2013. Biosens. Bioelectron. 41, 186-191. 